\title{
Fluctuation Dissipation Relations in Stationary States of Interacting Brownian Particles under Shear
}

\author{
Matthias Krüger and Matthias Fuchs \\ Fachbereich Physik, Universität Konstanz, 78467 Konstanz, Germany \\ (Received 11 July 2008; revised manuscript received 8 January 2009; published 31 March 2009)

\begin{abstract}
The fluctuation dissipation theorem is studied close to the glass transition in colloidal suspensions under steady shear. Shear breaks detailed balance in the many-particle Smoluchowski equation and gives response functions in the stationary state which are smaller at long times than estimated from the equilibrium fluctuation dissipation theorem. During the final shear-driven decay, an asymptotically constant relation connects response and fluctuations, restoring the form of the fuctuation dissipation theorem with, however, a ratio different from the equilibrium one.
\end{abstract}

PACS numbers: 64.70.P - , 05.70.Ln, 82.70.Dd, 83.60.Df

In thermal equilibrium, the response of a system to a small external perturbation follows directly from thermal fluctuations of the unperturbed system. This connection is the essence of the fluctuation dissipation theorem (FDT) which lies at the heart of linear response theory. In nonequilibrium systems, much work is devoted to understanding the general relation between fluctuation [correlator $C(t)$ ] and response [susceptibility $\chi(t)$ ] functions. It has been characterized by the fluctuation dissipation ratio (FDR) $X(t)$ defined via

$$
\chi(t)=-\frac{X(t)}{k_{B} T} \frac{\partial}{\partial t} C(t) .
$$

It is unity close to equilibrium $\left[X^{(e)}(t)=1\right]$ but deviates in nonequilibrium because the external perturbations act against nonvanishing currents [see Eq. (2) below]; FDRs quantify the currents and signal nonequilibrium [1].

Colloidal dispersions exhibit slow cooperative dynamics at high concentrations and form glasses. These metastable soft solids can easily be driven into stationary states far from equilibrium by shearing with already modest flow rates. Spin glasses driven by nonconservative forces were predicted to exhibit nontrivial FDRs in mean field models [2]. Such behavior was observed in detailed computer simulations of sheared supercooled liquids by Berthier and Barrat [3]. During the shear-induced relaxation, the FDR for particle motion perpendicular to the shear plane is different from unity but constant in time. This ratio was also found to be independent of the observable, which led to the notion of an effective temperature $X=T / T_{\text {eff }}$ describing the nonequilibrium state. Further simulations with shear also saw $T_{\text {eff }}>T$ [4-6], and recently $T_{\text {eff }}$ was connected to barrier crossing rates [7].

On the theoretical side, much effort has been made on different spin models, close to criticality. Universal FDRs were found under coarsening [8] and under shear [9], where, at the critical temperature, a value of $X=\frac{1}{2}$ was found in some cases. Yet the situation for structural glasses has not been clarified.
In this Letter, we investigate the FDT for colloidal suspensions close to a glass transition under steady shear starting from the $N$-particle Smoluchowski equation. While time-dependent correlation functions are calculated in the integration through transients (ITT) approach [10], which is based on mode coupling theory, the connection to the corresponding susceptibilities will be derived for the first time here. We show that equilibrium FDT is violated but can be restored in a well defined sense with a renormalized FDR at long times; however, the ratio depends on the variable, contradicting the notion of an effective temperature. Moreover, we establish a connection to the concept of a yield stress, which gives a scenario quite different from mean field spin glass [2].

$N$ spherical Brownian particles of diameter $d$, with bare diffusivity $D_{0}$, and interacting via internal forces $\mathbf{F}_{i}=$ $-\partial_{i} U, i=1, \ldots, N$, are dispersed in a solvent with a steady and homogeneous velocity profile $\mathbf{v}(\mathbf{r})=\boldsymbol{\kappa} \cdot \mathbf{r}$, with shear rate tensor $\boldsymbol{\kappa}=\dot{\gamma} \hat{\mathbf{x}} \hat{\mathbf{y}}$. Neglecting hydrodynamic interactions, the distribution of particle positions evolves according to the Smoluchowski equation [10]

$$
\partial_{t} \Psi(t)=\Omega \Psi(t), \quad \Omega=\sum_{i} \boldsymbol{\partial}_{i} \cdot\left[\boldsymbol{\partial}_{i}-\mathbf{F}_{i}-\boldsymbol{\kappa} \cdot \mathbf{r}_{i}\right]
$$

where $\Omega$ is the Smoluchowski operator and we have introduced dimensionless units for length, energy, and time: $d=k_{B} T=D_{0}=1$. The Smoluchowski operator for the system without shear $(\boldsymbol{\kappa}=\mathbf{0})$ and the flow part will be denoted $\Omega_{e}$ and $\delta \Omega=\Omega-\Omega_{e}$. We distinguish two timeindependent distributions: $\Omega_{e} \Psi_{e}=0$ without shear and $\Omega \Psi_{s}=0$ for the stationary system. Averages are $\langle\ldots\rangle$ and $\langle\ldots\rangle^{(\dot{\gamma})}$, respectively. Stationary correlation functions are $C_{a b}(t)=\left\langle\delta a^{*} e^{\Omega^{\dagger} t} \delta b\right\rangle^{(\dot{\gamma})}$, where $\Omega^{\dagger}$ is the adjoint operator obtained by partial integrations [10,11]; a fluctuation equals $\delta a=a-\langle a\rangle^{(\dot{\gamma})}$. Note that shear in Eq. (1) leads to a non-Hermitian eigenvalue problem [12]. The susceptibility $\chi_{a b}(t)$ describes the linear response of the stationary expectation value of $b$ to an external perturbation $h_{e}(t)$ shifting the internal energy $U$ to $U-a^{*} h_{e}(t)$ : 


$$
\langle b\rangle^{\left(\dot{\gamma}, h_{e}\right)}(t)-\langle b\rangle^{(\dot{\gamma})}=\int_{-\infty}^{t} d t^{\prime} \chi_{a b}\left(t-t^{\prime}\right) h_{e}\left(t^{\prime}\right)+\mathcal{O}\left(h_{e}^{2}\right) .
$$

One finds $\chi_{a b}(t)=\left\langle\sum_{i} \frac{\partial a^{*}}{\partial \mathbf{r}_{i}} \cdot \partial_{i} e^{\Omega^{\dagger} t} b\right\rangle^{(\dot{\gamma})}[11]$. In nonequilibrium, where detailed balance is broken and a nonzero stationary probability current $\mathbf{j}_{i}^{S}=\left[-\boldsymbol{\partial}_{i}+\mathbf{F}_{i}+\boldsymbol{\kappa} \cdot \mathbf{r}_{i}\right] \Psi_{s}$ exists, the equilibrium FDT is extended (with $\hat{j}_{i}^{\dagger}$ the adjoint of the current operator defined by $\mathbf{j}_{i}^{s}=\hat{j}_{i} \Psi_{s}$ ):

$$
\Delta_{\chi a b}(t)=\chi_{a b}(t)+\dot{C}_{a b}(t)=-\left\langle\sum_{i} \hat{j}_{i}^{\dagger} \cdot \frac{\partial a^{*}}{\partial \mathbf{r}_{i}} e^{\Omega^{\dagger}} b\right\rangle^{(\hat{\gamma})},
$$

and a deviation of the FDR $X_{a b}(t)=\frac{\chi_{a b}(t)}{-\bar{C}_{a t}(t)}$ from unity, the value close to equilibrium, arises. While Eq. (2) has been known since the work of Agarwal [11], we will analyze it for driven metastable (glassy) states and show that the additive correction $\Delta_{\chi_{a b}}(t)[13-15]$ leads to the nontrivial multiplicative correction, i.e., a constant FDR at long times. For simplicity, we will look at autocorrelations $(b=$ a) of $x$-independent fluctuations $\delta \Omega^{\dagger} a=0$, where the flow term in the current operator $\hat{j}_{i}^{\dagger}$ in (2) vanishes.

$\Psi_{s}$ is not known, and stationary averages are calculated via the ITT approach [10]: $\langle\ldots\rangle^{(\dot{\gamma})}=\langle\ldots\rangle+$ $\dot{\gamma} \int_{0}^{\infty} d s\left\langle\sigma_{x y} e^{\Omega 2^{\dagger}} \ldots\right\rangle$, where $\sigma_{x y}=-\sum_{i} F_{i}^{x} y_{i}$ is a microscopic stress tensor element. (Operators act on everything to the right, except for when marked differently by bracketing.) The ITT simplifies the following analysis because averages can now be evaluated in equilibrium, while otherwise nonequilibrium forces would be required [16]. Because of $\partial_{i} \Psi_{e}=F_{i} \Psi_{e}$, expression (2) vanishes in the equilibrium average. The remaining term is split into three pieces containing $\Omega^{\dagger}$ :

$$
\begin{aligned}
\Delta \chi_{a}(t)= & \frac{-\dot{\gamma}}{2} \int_{0}^{\infty} d s\left\langle\sigma _ { x y } e ^ { \Omega ^ { \dagger } s } \left[\Omega^{\dagger} a^{* \prime}-a^{*} \Omega^{\dagger}\right.\right. \\
& \left.\left.+\left(\Omega^{\dagger} a^{*}\right)\right] e^{\Omega^{\dagger}{ }^{\dagger}} a\right\rangle .
\end{aligned}
$$

We start with the first term in the square brackets (without the factor $\frac{1}{2}$ ) which can be integrated over $s$ directly, giving

$$
\dot{\gamma}\left\langle\sigma_{x y} \delta a^{*} e^{\Omega \iota^{\dagger}} \delta a\right\rangle=\left.\frac{\partial}{\partial t_{w}} C_{a}\left(t, t_{w}\right)\right|_{t_{w}=0},
$$

where from now on we consider fluctuations from equilibrium $\delta a=a-\langle a\rangle$ [the constant $\langle a\rangle$ cancels in (3)]. Intriguingly, in Eq. (4) the two-time correlator enters:

$$
C_{a}\left(t, t_{w}\right)=\left\langle\delta a^{*} e^{\Omega^{\dagger_{t}}} \delta a\right\rangle+\dot{\gamma} \int_{0}^{t_{w}} d s\left\langle\sigma_{x y} e^{\Omega^{\dagger_{s}}} \delta a^{*} e^{\Omega^{\dagger_{f}}} \delta a\right\rangle,
$$

where the rheometer has been shearing for a period $t_{w}$ before the correlation measurement is started. It is one of the central quantities in the spin-glass theory of aging [2]. While the transient correlator $C_{a}^{(t)}(t)=C_{a}(t, 0)=$ $\left\langle\delta a^{*} e^{\Omega^{\dagger}{ }^{\dagger}} \delta a\right\rangle$ describes the dynamics after switching on the rheometer, the stationary correlator $C_{a}(t)=C_{a}(t, \infty)$ is observed after waiting long enough; it measures fluctuations in the stationary state.

Our approximation for $\Delta_{\chi_{a}}(t)$ in Eq. (2) rests on the observation that it contains the product of a fluctuation $\delta a$ and the stationary current. We expect current fluctuations to always decay to zero, even in possible nonergodic situations, and thus search for a coupling of $\Delta_{\chi_{a}}(t)$ to derivatives of $C_{a}(t)$ as they cannot be nonergodic. Partial integration can be used to show $\left.\frac{\partial}{\partial t_{w}} C_{a}\left(t, t_{w}\right)\right|_{t_{w}=0}=$ $\dot{C}_{a}^{(t)}(t)-\left\langle\left(\Omega_{e}^{\dagger} a^{*}\right) e^{\Omega^{\dagger} t} \delta a\right\rangle$, where the latter term contains the equilibrium derivative $\Omega_{e}^{\dagger} a^{*}$. It is not conserved and decorrelates quickly as the particles lose memory of their initial motion even without shear. The latter term then is the time derivative of the equilibrium correlator $C_{a}^{(e)}(t)=$ $\left\langle\delta a^{*} e^{\Omega_{e}^{\dagger} t} \delta a\right\rangle$. A shear flow switched on at $t=0$ should make the particles forget their initial motion even faster, prompting us to use the approximation $e^{\Omega^{\boldsymbol{t}_{t}}} \approx$ $e^{\Omega_{e}^{\dagger} t} P e^{-\Omega \Omega_{e}^{\dagger} t} e^{\Omega \Omega^{\dagger} t}$, with projector $\left.P=\delta a\right\rangle\left\langle\delta a^{*} \delta a\right\rangle^{-1}\left\langle\delta a^{*}\right.$ in $\left\langle\left(\Omega_{e}^{\dagger} a^{*}\right) e^{\Omega^{\dagger} \dagger} \delta a\right\rangle$; it is then assured to decay faster than in equilibrium. This leads, together with an analogous approximation in $\left\langle\delta a^{*} e^{\Omega^{\dagger}} \delta a\right\rangle$, to

$$
\left.\frac{\partial}{\partial t_{w}} C_{a}\left(t, t_{w}\right)\right|_{t_{w}=0} \approx \dot{C}_{a}^{(t)}(t)-\dot{C}_{a}^{(e)}(t) \frac{C_{a}^{(t)}(t)}{C_{a}^{(e)}(t)} .
$$

The last term in (6) will be identified as the short time derivative of $C_{a}^{(t)}$, connected with the shear-independent decay, while $\left.\frac{\partial}{\partial t_{w}} C_{a}\left(t, t_{w}\right)\right|_{t_{w}}=0$ will turn out to be the long time derivative of $C_{a}^{(t)}$, connected with the final sheardriven decay. It captures the additional dissipation provided by the coupling to the stationary current in Eq. (2).

Now the difference between the stationary and the transient correlators needs to be found via Eq. (5):

$$
C_{a}(t)-\left.C_{a}^{(t)}(t) \approx \int_{0}^{\infty} d s \frac{\left\langle\sigma_{x y} e^{\Omega \Omega^{\dagger} s} \sigma_{x y}\right\rangle}{\left\langle\sigma_{x y} \sigma_{x y}\right\rangle} \frac{\partial C_{a}\left(t, t_{w}\right)}{\partial t_{w}}\right|_{t_{w}=0},
$$

where we used $t_{w}=\infty$, and factorized the appearing twotime average with the projector $\left.\sigma_{x y}\right\rangle\left\langle\sigma_{x y} \sigma_{x y}\right\rangle^{-1}\left\langle\sigma_{x y}\right.$. A small parameter $\tilde{\sigma} \equiv \dot{\gamma} \int_{0}^{\infty} d s\left\langle\sigma_{x y} \exp \left(\Omega^{\dagger} s\right) \sigma_{x y}\right\rangle /\left\langle\sigma_{x y} \sigma_{x y}\right\rangle$ arises whose numerator is the stationary shear stress measured in "flow curves" as a function of shear rate [10]. For hard spheres, the instantaneous shear modulus $\left\langle\sigma_{x y} \sigma_{x y}\right\rangle$ diverges, giving formally $\tilde{\sigma}=0$ and that the transient and stationary correlators agree. In recent simulations of density fluctuations of soft spheres [17], the difference between the two correlators was found to be largest at intermediate times, and $C_{a}(t) \leq C_{a}^{(t)}(t)$ was observed. Both properties are fulfilled by Eq. (7).

After the discussion of the first term in (3), we turn to the correction containing the last two terms in (3). It has a vanishing initial value, and, in a mode coupling approximation in the ITT for the case of density fluctuations, the 
two terms also almost cancel each other at long times, making their sum a small correction. Here we proceed by ignoring it until a future presentation. We hence find

$$
\chi_{a}(t) \approx-\dot{C}_{a}(t)+\frac{1}{2}\left(\dot{C}_{a}^{(t)}(t)-\dot{C}_{a}^{(e)}(t) \frac{C_{a}^{(t)}(t)}{C_{a}^{(e)}(t)}\right) .
$$

In the limit of small shear rates for glassy states, the correlators exhibit two separated relaxation steps $[10,18]$. During the shear-independent relaxation onto the plateau of height given by the nonergodicity parameter $f_{a}$, we have $C_{a}^{(t)}(t) \approx C_{a}^{(e)}(t)$, and the equilibrium FDT holds. During the shear-induced final relaxation from $f_{a}$ down to zero, i.e., for $\dot{\gamma} \rightarrow 0$, and $t \rightarrow \infty$, with $t \dot{\gamma}=$ const, the correlator without shear stays on the plateau and its derivative is negligible. A nontrivial FDR follows.

$$
\lim _{\dot{\gamma} \rightarrow 0} \chi_{a}(t)= \begin{cases}-\dot{C}_{a}(t) & \dot{\gamma} t \ll 1, \\ -\dot{C}_{a}(t)+\frac{1}{2} \dot{C}_{a}^{(t)}(t) & \dot{\gamma} t=\mathcal{O}(1) .\end{cases}
$$

It is interesting to note that, approximating stationary and transient correlators to be equal [10] $C_{a}^{(t)}(t) \approx C_{a}(t)$, we find $\chi_{a}(t)=-\frac{1}{2} \dot{C}_{a}(t)$ for long times. The FDR in this case takes the universal value $\lim _{\dot{\gamma} \rightarrow 0} X_{a}(t \rightarrow \infty)=$ $\hat{X}^{\text {(univ) }}(\dot{\gamma} t)=\frac{1}{2}$, independent of $a$. This is in good agreement with the findings in Ref. [3]. The initially additive correction in Eq. (2) hence turns then into a multiplicative one, which does not depend on rescaled time during the complete final relaxation process.

For a more precise investigation of the FDR, we have to consider the difference between the transient and the stationary correlators in Eq. (7). We turn to the schematic $F_{12}^{(\dot{\gamma})}$ model of the ITT [19], which has repeatedly been used to investigate the dynamics of quiescent and sheared dispersions and which provides excellent fits to the flow curves from large scale simulations [20]. It provides a normalized transient correlator $C^{(t)}(t)$, as well as a quiescent one, representing coherent, i.e., collective, density fluctuations. The corresponding stationary correlator $C$ is calculated in a second step via Eq. (7). Figure 1 shows the resulting $\chi$ together with $C$ for a glassy state at different shear rates. For short times the equilibrium FDT is valid, while for long times the susceptibility is smaller than expected from the equilibrium FDT; this deviation is qualitatively similar for the different shear rates. For the smallest shear rate, we also plot $\chi$ calculated by Eq. (8) with $\dot{C}_{a}^{(t)}$ replaced by $\dot{C}_{a}$, from which the universal $\hat{X}^{(\text {univ) }}(\dot{\gamma} t)=\frac{1}{2}$ follows. In the parametric plot (Fig. 2), this leads to two perfect lines with slopes -1 and $-\frac{1}{2}$ connected by a sharp kink at the nonergodicity parameter $f$. For the other (realistic) curves, this kink is smoothed out, but the long time part is still well described by a straight line; i.e., the FDR is still almost constant during the final relaxation process. We predict a nontrivial time-independent FDR $\hat{X}_{a}(\dot{\gamma} t)=$ const if $C_{a}^{(t)}$ [and with Eq. (7) also $C_{a}$ ] decays exponentially for long times, because $\Delta_{\chi_{a}}$ then decays exponentially with the

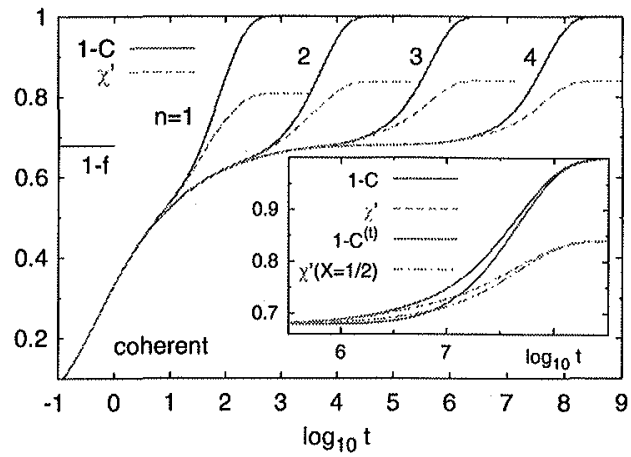

FIG. 1 (color online). $\quad C(t)$ from the $F_{12}^{(\dot{\gamma})}$ model [19] and $\chi(t)$ via $\mathrm{Eq}$. (8) for a glassy state $\left(\varepsilon=10^{-3}\right)$ and $\dot{\gamma}=10^{-2 n}$, with $n=1, \ldots, 4$. Shown are integrated correlation $1-C(t)$ and response $\chi^{\prime}(t)=\int_{0}^{t} \chi\left(t^{\prime}\right) d t^{\prime}$. The inset shows additionally the normalized transient correlator $C^{(t)}$ for comparison and the $\hat{X}^{\text {(univ) }}=\frac{1}{2}$ susceptibility for $\dot{\gamma}=10^{-8}$.

same exponent. The line cuts the FDT line below $f$ for $\dot{\gamma} \rightarrow$ 0 . All of these findings are in excellent agreement with the data in Ref. [3]. The FDR itself is of interest also, as a function of time (inset in Fig. 2). A sharp transition from 1 to $\frac{1}{2}$ is observed when $C^{(t)} \approx C$ is approximated, which takes place at $\dot{\gamma} t \approx 10^{-3}$, when the FDT violation is still invisible in Fig. 1. For the realistic curves, this transition happens two decades later. The huge difference is strikingly not apparent in the parametric plot.

Figure 3 shows the long time FDR as a function of shear rate for different densities above and below the glass transition, determined via fits to the parametric plot in the interval [0:0.1]. In the glass $X(t \rightarrow \infty)$ is nonanalytic, while it goes to unity in the fluid as $\dot{\gamma} \rightarrow 0$, where we verified that the FDT violation starts quadratic in $\dot{\gamma}$ as is to be expected due to symmetries. We can also study the variable dependence of the FDR, for which we consider incoherent, i.e., single, particle fluctuations [19] which

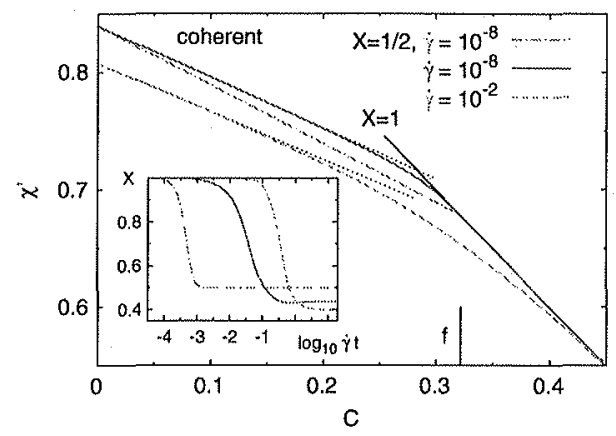

FIG. 2 (color online). Parametric plot of correlation $C(t)$ versus response $\chi^{\prime}(t)=\int_{0}^{t} \chi\left(t^{\prime}\right) d t^{\prime}$ for a glassy state $\left(\varepsilon=10^{-3}\right)$ from the $F_{12}^{(\dot{\gamma})}$ model [19] together with a constant nontrivial FDR (straight lines) at long times. The vertical solid line marks the plateau $f$. The inset shows the FDR $X(t)$ as a function of strain for the same susceptibilities. 


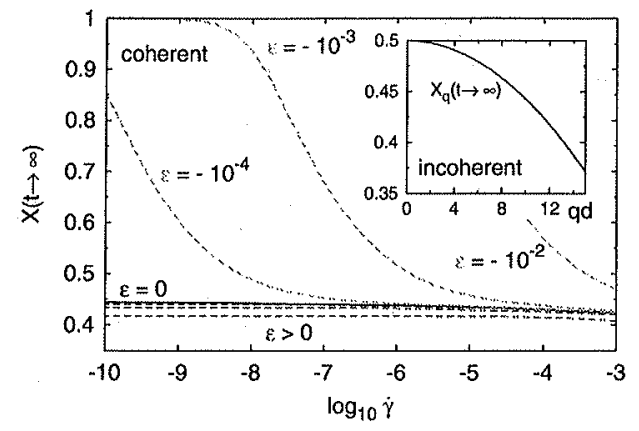

FIG. 3 (color online). Long time FDR as a function of shear rate for glasses $(\varepsilon \geq 0)$ and liquids $(\varepsilon<0)$ in the $F_{12}^{(\dot{\gamma})}$ model [19], when approaching the transition for $\varepsilon= \pm 10^{\{-2,-3,-4\}}$. Inset: $\lim _{j \rightarrow 0} X_{q}(t \rightarrow \infty)$ versus wave vector $q$ for incoherent density fluctuations at the critical density $(\varepsilon=0)$ [19].

were most extensively studied in Ref. [3]. The FDR is isotropic in the plane perpendicular to the shear direction but not independent of wave vector $q$, contradicting the idea of an effective temperature as proposed in Refs. [2,3] and others (see inset in Fig. 3).

That Eq. (8) is nevertheless not in contradiction to the data in Ref. [3] can be seen by direct comparison to their Fig. 11. For this, we need the quiescent as well as the transient correlator as input. $C_{q}^{(e)}$ has been measured in Ref. [18] suggesting that it can be approximated by a straight line beginning on the plateau of $C_{\mathrm{q}}(t)$. In Fig. 4 , we show the resulting susceptibilities. There is no adjustable parameter, when $C_{\mathrm{q}}^{(t)} \approx C_{\mathrm{q}}$ is taken; for the other curve, we calculated $C_{\mathrm{q}}^{(t)}(t)$ from Eq. (7) using $\tilde{\sigma}=0.01$ (in Lennard-Jones units) as the fit parameter. The agreement is striking. In the inset, we show the original $C_{\mathbf{q}}$ from Ref. [3] together with our construction of $C_{q}^{(e)}$ and the

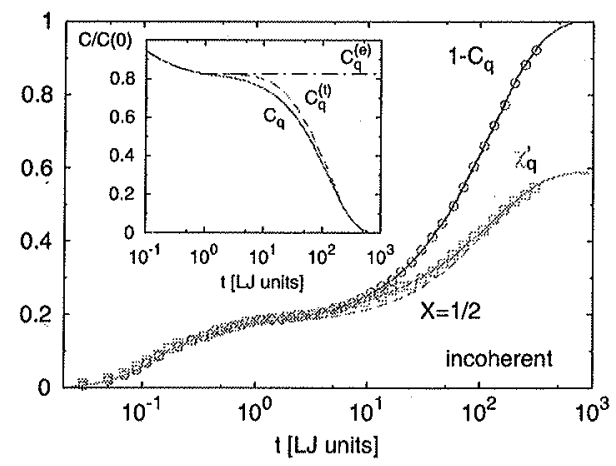

FIG. 4 (color online). Comparison to simulation data for incoherent density fluctuations in the neutral direction (wave vector $\left.\mathbf{q}=7.47 \mathbf{e}_{z}\right)$ at temperature $T=0.3\left(T_{c}=0.435\right)$ and $\dot{\gamma}=$ $10^{-3}$. Circles and squares are the data (including units) from Fig. 11 in Ref. [3(a)]; lines are $1-C_{q}$ from Fig. 8 in Ref. [3(a)] and the response $\chi_{\mathfrak{q}}^{\prime}(t)=\int_{0}^{t} \chi_{\mathrm{q}}\left(t^{\prime}\right) d t^{\prime}$ calculated via Eq. (8). The dashed line shows $\chi_{\mathbf{q}}^{\prime}$ with approximation $C_{\mathbf{q}}^{(t)} \approx C_{\mathbf{q}}$. The inset shows the different correlators; see the main text. calculated $C_{\mathbf{q}}^{(t)}$, which appears very reasonable compared with recent simulation data on $C_{a}\left(t, t_{w}\right)$ [17].

In summary, shear flow drives metastable Brownian dispersions to a stationary nonequilibrium state with a multiplicative renormalization of the FDR at long times, which is (almost) independent of rescaled time. It nearly agrees for variables not advected by flow and takes the universal value $\hat{X}_{a}(\dot{\gamma} t)=\frac{1}{2}$. in glasses at small shear rates in the leading approximation. Corrections arise from the difference of the stationary to the transient correlator and depend on the considered variable. They alter $\hat{X}_{a}$ to values $\hat{X}_{a} \leq \frac{1}{2}$ in the glass. We show a new testable connection between $\Delta \chi_{a}$ and $C_{a}\left(t, t_{w}\right)$ [see Eq. (4)].

The derived FDRs characterize the shear-driven relaxation at long times, which, according to the ITT approach, is also the origin of a (dynamic) yield stress in shear molten glass [10]. This view captures extended simulations $[3,20]$ and broadband experiments [21], establishing shear molten glass as a model for investigating nonequilibrium. Open questions concern addressing the concept of an effective temperature developed in mean field spin models.

We thank J.-L. Barrat, M. E. Cates, and P. IIg for helpful discussions. M. K. was supported by the DFG in IRTG 667 .

[1] A. Crisanti and F. Ritort, J. Phys. A 36, R181 (2003).

[2] L. Berthier, J.-L. Barrat, and J. Kurchan, Phys. Rev. E 61, 5464 (2000).

[3] (a) L. Berthier and J.-L. Barrat, J. Chem. Phys. 116, 6228 (2002); (b) Phys. Rev. Lett. 89, 095702 (2002).

[4] C. S. O'Hern, A. J. Liu, and S. R. Nagel, Phys. Rev. Lett. 93, 165702 (2004).

[5] T. K. Haxton and A. J. Liu, Phys. Rev. Lett. 99, 195701 (2007).

[6] F. Zamponi, G. Ruocco, and L. Angelani, Phys. Rev. E 71, 020101(R) (2005).

[7] P. Ilg and J.-L. Barrat, Europhys. Lett. 79, 26001 (2007).

[8] C. Godrèche and J. M. Luck, J. Phys. A 33, 1151 (2000).

[9] F. Corberi et al., J. Phys. A 36, 4729 (2003).

[10] M. Fuchs and M.E. Cates, Phys. Rev. Lett. 89, 248304 (2002); J. Phys. Condens. Matter 17, S1681 (2005).

[11] G. S. Agarwal, Z. Phys. 252, 25 (1972).

[12] R. Graham, Z. Phys. B, Condens. Matter 40, 149 (1980).

[13] V. Blickle et al., Phys. Rev. Lett. 98, 210601 (2007).

[14] T. Speck and U. Seifert, Europhys. Lett. 74, 391 (2006).

[15] T. Harada and S.-i. Sasa, Phys. Rev. Lett. 95, 130602 (2005).

[16] G. Szamel, Phys. Rev. Lett. 93, 178301 (2004).

[17] J. Zausch et al., J. Phys. Condens. Matter 20, 404210 (2008).

[18] F. Varnik, J. Chem. Phys. 125, 164514 (2006).

[19] See EPAPS Document No. E-PRLTAO-102-075915 for details on known ITT models. For more information on EPAPS, see http://www.aip.org/pubservs/epaps.html.

[20] F. Varnik and O. Henrich, Phys. Rev. B 73, 174209 (2006).

[21] M. Siebenbürger et al., arXiv:0810.3551 [J. Rheol. (N.Y.) (to be published)] 